\title{
Untersuchungen über die in den Pflanzen vorkommenden Betaine.
}

\author{
III. Mitteilung. ${ }^{1}$ ) \\ Von
}

E. Schulze und G. Trier.

(Aus dem agrikulturchemischen Laboratorium der Eidgenössischen Technischen Hochschule in Zürich.)

(Der Redaktion zugegangen am 1. Mai 1912.)

In der kurzen Zeit seit Erscheinen unserer letzten Mitteilung ${ }^{1}$ ) ist die Kenntnis der in Pflanzen vorkommenden Betaine in mehrfacher Hinsicht erweitert worden. Zunächst ist nachzutragen, daß sich das Hypaphorin nach der Untersuchung von van Romburgh und Barger ${ }^{2}$ ) tatsächlich als das Betain des Tryptophans erwiesen hat. Dann haben Barger und Ewins ${ }^{3}$ ) die sehr interessante Tatsache festgestellt, daß die im Jahre 1909 von Tanret $\left.{ }^{4}\right)$ im Mutterkorn aufgefundene schwefelhaltige Base, das Ergothionin, $\mathrm{C}_{9} \mathrm{H}_{15} \mathrm{O}_{2} \mathrm{~N}_{3} \mathrm{~S}$, ein Histidinbetain ist, das wahrscheinlich im Imidazolkern das Schwefelatom gebunden enthält. Mit Eisenchlorid behandelt, gibt das Ergothionin Histidinbetain, eine Verbindung, von der Barger und Ewins annehmen, $\mathrm{da} B$ sie auch in der Natur auftreten könnte. Nun ist von F. Kutscher ${ }^{5}$ ) eine Base aus Champignonextrakt erhalten worden, die dieser Forscher als Trimethylhistidin angesprochen hat, und kürzlich ist die offenbar gleiche Verbindung auch im hiesigen Laboratorium von C. Reuter ${ }^{6}$ ) aus dem alkoholischen

1) II. Mitteilung siehe Diese Zeitschrift, Bd. 76, S. 258.

2) Transactions of the Chemical Society, Bd. 99, S. 2068.

3) Transactions of the Chemical Society, Bd. 99, S. 2336.

4) Compt. rend. de l'Acad. des sciences, Bd. 149, S 222. - Journ. Pharm. Chim., Bd. 30, S. 145.

5) Zeitschrift f. Unters. d. Nahrungs- u. Genußmittel, Bd. 21, S. 535.

6) Diese Zeitschrift, Bd. 78, S. 201. Siehe dort auch die Bemerkungen zur Frage über die Identität dieser Verbindung mit dem von Barger und $\mathrm{Ew}$ ins erhaltenen Histidinbetain.

Hoppe-Seyler's Zeitschrift f. physiol. Chemie. LXXIX. 
Extrakt von Steinpilzen(Boletus edulis) isoliert worden. Ferner ist im hiesigen Laboratorium gefunden worden, ${ }^{1}$ ) daß das Stachyjdrin auch in der optisch aktiven, linksdrehenden Form auftritt. Sodann ist hier das von uns kürzlich entdeckte Betonicin zu nennen, eine Base, der wir die Formel $\mathrm{C}_{7} \mathrm{H}_{13} \mathrm{NO}_{3}$ zuwiesen. Dagegen ist die Existenz einer anderen betainartigen Base von komplizierterem Bau in Frage gestellt worden, da bei Untersuchung ${ }^{1}$ ) von «dalmatinischem Insektenpulver » an Stelle des von Marino-Zucco beschriebenen Chrysanthemins ${ }^{2}$ ) ein Gemisch von Basen gefunden wurde, aus welchem Cholin und Stachydrin gewonnen werden konnten.

In Fortsetzung unserer Untersuchungen sind wir nun auf mehrere neue Basen gestoßen, die wenigstens zum Teil ebenfalls den Betainen zugezählt werden müssen. Es hat sich nämlich herausgestellt, daß das Betonicin, von welchem in der letzten Abhandlung schon die Rede war, von allem Anscheine nach isomeren Basen begleitet wird und daß solche Basen, die ihrer Zusammensetzung nach dem Dimethylbetain des Oxyprolins entsprechen, in mehreren Pflanzen auftreten. Wir fanden solche Verbindungen in Betonica officinalis und in Stachys silvatica. Diese neuen Verbindungen lassen sich verhältnismäßig leicht von den schon bekannten Betainen abtrennen (in Betonica officinalis wurde Stachydrin, in Stachys silvatica Trigonellin nachgewiesen), da sie im Gegensatze zu diesen als freie Basen in absolutem Alkohol sehr schwer löslich sind. Hingegen gelingt eine vollkommene Trennung der einzelnen «Betonicinbasen*, wie wir sie der Kürze halber nennen wollen, voneinander nicht leicht. Näher beschrieben wurde bis jetzt daher nur jene als Betonicin bezeichnete Verbindung, die sich am leichtesten isolieren ließ, da ihr salzsaures Salz in absolutem Alkohol in der Kälte am wenigsten löslich ist. Falls es sich hier tatsächlich um Verbindungen handelt, die im gleichen Verhältnis zu Eiweißspaltungsprodukten stehen wie Betain, Stachydrin und Hypaphorin, könnte die nähere Untersuchung dieser Basen uns

1) K. Yoshimura u. G. Trier, Diese Zeitschrift, Bd. 77, S. 290.

2) Siehe unsere Bemerkung, Diese Zeitschrift, Bd. 67, S. 47. 
eventuell zur Kenntnis noch unbekannter Aminosäuren der Eiweißst offe führen.

Bei der Untersuchung größerer Mengen junger Wickenpflanzen erhielten wir neben Betain $\mathrm{C}_{5} \mathrm{H}_{11} \mathrm{NO}_{2}$ in der sogenannten Betainfraktion eine Verbindung (oder ein Gemenge von Verbindungen), welche den aus Labiaten gewonnenen *Betonicinbasen in vielen Punkten entsprach. Doch zeigte es sich, daß die freien Verbindungen sich in wesentlichen Eigenschaften von den «Betonicinbasen» unterschieden, und daß ihre Betainnatur vorläufig zweifelhaft erscheint.

Im folgenden beschreiben wir einige Versuche mit den Verbindungen, die aus jungen Pflanzen von Vicia sativa und aus dem Kraute von Betonica officinalis erhalten wurden.

Die nähere Untersuchung dieser Verbindungen, die noch einige Zeit in Anspruch nehmen wird, und ihren Vergleich mit synthetischen Präparaten aus den Produkten von Eiweißhydrolysen behalten wir uns vor.

Untersuchungen an jungen Wickenpflanzen (Vicia sativa).

Durch frühere Untersuchungen von E. Schulze und seinen Mitarbeitern war das Betain $\mathrm{C}_{5} \mathrm{H}_{11} \mathrm{NO}_{2}$ bei Vicia sativa sowohl in reifen, wie in unreifen Samenkörnern, in etiolierten Keimpflanzen, in Stengeln und Blättern normaler Pflanzen und in unreifen Samenhülsen nachgewiesen worden. In einer zu Anfang des Jahres 1911 ausgeführten Untersuchung fanden wir, daß eine aus Wickenpflanzen gewonnene «Betainfraktion» deutliche Pyrrolreaktion gab; doch verschwand diese Reaktion nach weiterer Reinigung der Salze. Es stellte sich später heraus, daß die, die Pyrrolreaktion gebende, Substanz beim Aufstreichen der Salze auf eine Tonplatte in diese gegangen war.

Im vergangenen Sommer verarbeiteten wir eine größere Menge junger Wickenplanzen. Die Pflanzen hatten eine Höhe von $35-40 \mathrm{~cm}$ über dem Boden erreicht. Sie kamen ohne Wurzeln zur Verwendung. Es wurden etwa $11 \mathrm{~kg}$ (lufttrocken) verarbeitet. Sie wurden mit $75 \%$ igem Alkohol extrahiert und die 
Extrakte zunächst nach dem wiederholt beschriebenen Verfahren behandelt. Die bei Zerlegung des zweiten Phosphorwolframsäureniederschlags erhaltene Lösung der salzsauren Salze wurde bis zur Krystallisation eingedunstet, die Masse sodann mit kaltem Alkohol behandelt, wobei salzsaures Betain, gemengt mit etwas Chlorkalium, zurückblieb. Der nach wiederholter Behandlung mit absolutem Alkohol von schwer löslichen salzsauren Salzen möglichst getrennte Anteil wurde dann in sodaalkalischer Lösung mit Kaliumperjodid gefällt, dieser, das Cholin enthaltende Niederschlag beseitigt und das Filtrat nach der Vorschrift von Stanĕk wieder in salzsaure Salze verwandelt. Sodann wurden die Salze in Alkohol gelöst und mit alkoholischer Sublimatlösung gefällt. Die Quecksilbersalze wurden aus Wasser umkrystallisiert, in mehrere Fraktionen zerlegt und jede mit Schwefelwasserstoff zersetzt. Die ersten Fraktionen der regenerierten salzsauren Salze krystallisierten nach vollständigem Eindunsten und Stehenlassen im Exsikkator. Die einzelnen Fraktionen wurden nach vollständiger Trocknung wiederholt mit kaltem absolutem Alkohol behandelt und von ungelöst gebliebenen Anteilen abfiltriert. Die nach dem Abdunsten des Alkohols erhaltenen Fraktionen gaben mit Goldchloridlösung ölige, ungemein schwer lösliche Fällungen, die nicht zur Krystallisation zu bringen waren und sich für die Analyse nicht eigneten. Nur die erste und größte Fraktion der Quecksilbersalze lieferte Goldsalze, die nach Abtrennung der am schwersten Iöslichen Anteile gut krystallisierten und deren Goldwert, wie die folgenden Bestimmungen zeigen, durch alle Fraktionen ziemlich konstant blieb.

1. Krystallisation

$0,3050 \mathrm{~g}$ Goldsalz gab $0,1208 \mathrm{~g} \mathrm{Au}=\mathbf{3 9 , 6 1} \% \mathrm{Au}$.

2. Krystallisation

$0,3852 \mathrm{~g}$ Goldsalz gab $0,1527 \mathrm{~g} \mathrm{Au}=\mathbf{3 9 , 6 4} \% \mathrm{Au}$.

3. Krystallisation

$0,1773 \mathrm{~g}$ Goldsalz gab $0,0718 \mathrm{~g} \mathrm{Au}=\mathbf{4 0 , 5 0} \% \mathrm{Au}$.

4. Krystallisation

$0,1331 \mathrm{~g}$ Goldsalz gab $0,0530 \mathrm{~g} \mathrm{Au}=\mathbf{3 9 , 8 6} \% \mathrm{Au}$.

Alle Fraktionen gaben intensive Pyrrolreaktion. Die Goldsalze zeigten alle ganz ähnliche Zersetzungspunkte, die inner- 
halb jener Temperaturen lagen, die auch bei den Betainen der Pyrrolidingruppe (Stachydrin, Betonicin) beobachtet wurden.

Die aus den Goldsalzen regenerierten salzsauren Salze verhielten sich aber verschieden. Während die Salze aus den ersten Fraktionen (schwerer lösliche Goldsalze) krystallisierten, blieben die Salze der späteren Fraktionen sirupös.

Die mittels feuchten Silberoxyds erhaltenen freien Verbindungen waren in Alkohol schwer löslich.

Eine etwas größere Menge wurde wieder analysiert und dann in Form ihres salzsauren Salzes und als freie Base polarisiert.

$1,1242 \mathrm{~g}$ Goldsalz gaben $0,4444 \mathrm{~g} \mathrm{Au}=39,53 \% \mathrm{Au}$.

Das daraus dargestellte salzsaure Salz zeigte im SoleilVentzkeschen Apparat eine Drehung von $+0,6^{\circ} \mathrm{S} .-\mathrm{V}$. bei $20^{\circ}$ (in $8,1 \mathrm{ccm} \mathrm{H}_{2} \mathrm{O}$ im $1 \mathrm{dm}-\mathrm{Rohr}$ ).

$$
\alpha_{\mathrm{D}}^{20^{\circ}}+3,8^{\circ} \text {. }
$$

Die daraus erhaltenen freien Basen gaben ebenfalls nur eine sehr geringe Rechtsdrehung.

Die bisher beschriebenen Beobachtungen stimmen sehr gut mit den unten beschriebenen Beobachtungen an den Basen aus der Betonica überein. In einem wesentlichen Punkte sind aber beide Gruppen verschieden. Bei der Darstellung der freien Basen aus Vicia wurden beträchtliche Mengen Silberoxyd gelöst und ein in Wasser ziemlich schwer lösliches, schön krystallisierendes Silbersalz beobachtet.

Die freien Basen (so dürfen wir wohl durch Phosphorwolframsäure fällbare Verbindungen, die gut krystallisierende Aurate geben, nennen) vermögen auch Kupferoxyd mit blauer Farbe aufzulösen. Das bis jetzt nicht krystallisiert erhaltene Kupfersalz ist in Alkohol ganz unlöslich. Sie sind ferner in Wasser, aus welchen sie in feinen Nadeln krystallisieren, nicht ganz leicht löslich und reagieren deutlich sauer. Wir haben es hier also offenbar mit Aminosäuren (die möglicherweise am Stickstoff Methylgruppen tragen), aber allem Anschein nach nicht mit quaternären Basen, nicht mit Betainen zu tun. 


\section{Untersuchung des getrockneten Krautes von Betonica officinalis.}

Die Beobachtung, daß die neuen betainartigen Basen aus den Labiaten Stachys silvatica und Betonica officinalis im Gegensatz zu den anderen Pflanzenbetainen in Alkohol sehr schwer löslich sind, benutzend, änderten wir für ihre Gewinnung und Abtrennung vom Cholin und anderen Basen unsere gewöhnliche Darstellungsart in entsprechender Weise ab. Darüber soll später im Zusammenhang berichtet werden.

Aus $30 \mathrm{~kg}$ getrockneter «Herba betonicae» wurde eine ansehnliche Ausbeute an diesen Basen gewonnen.

Eine Probe wurde mit Goldlösung gefällt und die einzelnen Fraktionen analysiert, indem die Mutterlaugen eingedunstet und so stets neue Krystallisationen erhalten wurden.

\section{Krystallisation}

a) $0,1627 \mathrm{~g}$ Goldsalz gaben $0,0628 \mathrm{~g} \mathrm{Au}=\mathbf{3 8 , 6 0} \% \mathrm{Au}$.

b) $0,1826 \mathrm{~g}$ Goldsalz gaben $0,0718 \mathrm{~g} \mathrm{Au}=\mathbf{3 9 , 3 2} \% \mathrm{Au}$.

2. Krystallisation

$0,4027 \mathrm{~g}$ Goldsalz gaben $0,1594 \mathrm{~g} \mathrm{Au}=\mathbf{3 9 , 5 8} \% \mathrm{Au}$.

3. Krystallisation

$0,2919 \mathrm{~g}$ Goldsalz gaben 0,1152 $\mathrm{g} \mathrm{Au}=\mathbf{3 9 , 4 7 \%} \mathrm{Au}$.

4. Krystallisation

$0,1677 \mathrm{~g}$ Goldsalz gaben $0,0664 \mathrm{~g} \mathrm{Au}=\mathbf{3 9 , 6 0} \% \mathrm{Au}$.

Für $\mathrm{C}_{7} \mathrm{H}_{13} \mathrm{NO}_{3} \cdot \mathrm{HClAuCl}_{3}$ berechnet $39,53 \% \mathrm{Au}$.

Die freien Basen krystallisieren aus verdünntem Alkohol in schönen Krystallen, die allmählich verwittern. Die Krystalle enthalten 1 Molekül $\mathrm{H}_{2} \mathrm{O}$.

Die Krystalle wurden durch Abpressen zwischen Filterpapier von anhaftender Feuchtigkeit (wahrscheinlich nicht ganz vollkommen) befreit.

$0,2091 \mathrm{~g}$ Substanz verloren beim Trocknen bei $105^{\circ}$ $0,0231 \mathrm{~g} \mathrm{H}_{2} \mathrm{O}=\mathbf{1 1 , 0 5} \% \mathrm{H}_{2} \mathrm{O}$.

Für eine Verbindung $\mathrm{C}_{7} \mathrm{H}_{13} \mathrm{NO}_{3}+\mathrm{H}_{2} \mathrm{O}$ berechnet sich $10,17 \% \mathrm{H}_{2} \mathrm{O}$.

Eine Probe der getrockneten Substanz gab folgende Werte bei der Elementaranalyse: 


\section{$0,2088 \mathrm{~g}$ Substanz gab $0,4028 \mathrm{~g} \mathrm{CO}_{2}$ und $0,1514 \mathrm{~g} \mathrm{H}_{\mathbf{2}} \mathrm{O}$.} $0,2152 \mathrm{~g}$ Substanz gab $17,60 \mathrm{ccm} \mathrm{N}$ bei $17^{\circ}$ C. und $730 \mathrm{~mm}$.

Für $\mathrm{C}_{7} \mathrm{H}_{13} \mathrm{NO}_{3}$ berechnet:

$$
\begin{array}{cr}
\mathrm{C} & 52,79 \% \\
\mathrm{H} & 8,23 \% \\
\mathrm{~N} & 8,80 \%
\end{array}
$$

Gefunden: $52,61 \%$ $8,11 \%$ $9,06 \%$.

Während die so erhaltenen Analysenzahlen auf eine einheitliche Verbindung von der Zusammensetzung eines. Dimethylbetains des Oxyprolins hindeuteten, ließ sich doch leicht zeigen, daß es sich um ein Gemisch handle. Die salzsauren Salze dieser Verbindungen hatten eine ungleiche Löslichkeit in Alkohol und die so durch fortgesetzte Trennung erhaltenen Präparate zeigten entgegengesetztes optisches Drehungsvermögen. Die in Alkohol am schwersten löslichen Anteile, welche dem aus der ersten Darstellung nur in sehr kleiner Ausbeute erhaltenen Betonicin entsprechen müssen, gaben:

$0,5522 \mathrm{~g}$ Chlorhydrat in $6 \mathrm{ccm} \mathrm{H}_{2} \mathrm{O}$ bei $20^{\circ} \mathrm{C}$. im $1 \mathrm{dm}-$ Rohr $-6,35^{\circ}$ S.V.

$$
\alpha_{D}^{20}=-23,73^{\circ} \text {. }
$$

Das als salzsaures Betonicin bezeichnete Präparat aus Stachys silvatica ${ }^{1}$ ) drehte rechts und $z$ war gaben:

$1,0300 \mathrm{~g}$ in $6,5 \mathrm{ccm} \mathrm{H}_{2} \mathrm{O}$ bei $17^{\circ}+9,9^{\circ} \mathrm{S}$.V. im $1 \mathrm{dm}-\mathrm{Rohr}$

$$
\alpha_{\mathrm{D}}=+21,5^{\circ} \text {. }
$$

Es könnten optische Isomere vorliegen, da vorläufig nicht entschieden werden konnte, wie weit die Trennung von den anderen begleitenden Basen gelungen ist. Ein Teil der salzsauren Salze aus dem Betaingemisch ist zerfließlich. Auch dieser Teil gibt Goldsalze, deren Goldgehalt mit dem eines Oxyprolinbetainaurats übereinstimmt.

$0,1675 \mathrm{~g}$ Goldsalz gaben $0,0662 \mathrm{~g} \mathrm{Au}=\mathbf{3 9 , 5 2} \% \mathrm{Au}$. Für $\mathrm{C}_{7} \mathrm{H}_{13} \mathrm{NO}_{3} \cdot \mathrm{HClAuCl}_{3}$ berechnet 39,53\% Au.

Alle Fraktionen gaben starke Pyrrolreaktion.

Das Gemisch der Betaine, wie es aus der Betonica erhalten worden ist, zeigt eine geringe Rechtsdrehung. Ihre salzsauren Salze drehten ebenfalls schwach rechts.

1) Siehe die II. Mitteilung, Diese Zeitschrift, Bd. 76, S. 272. 
Die freien Basen zeigten in etwa 10\% iger Lösung für $\alpha_{D}=+2,10$. Die salzsauren Salze in etwa $15 \%$ iger Lösung $\alpha_{D}=+3,7^{\circ}$. Zum Unterschiede von den sonst so ähnlichen Verbindungen aus Vicia sativa reagieren Betonicin und seine Begleiter neutral und vermögen, wie andere Betaine, weder Silberoxyd noch Kupferoxyd aufzulösen.

Durch die hier mitgeteilten Versuche haben wir unsere Angabe bestätigen können, wonach in gewissen Labiaten betainartige Basen von der Zusammensetzung eines Dimethylbetains des Oxyprolins auftreten. Es hat sich aber gezeigt, daß die Sachlage dadurch komplizierter wird, daß die schon früher beschriebene und als Betonicin bezeichnete Verbindung von isomeren oder doch sehr ähnlichen Basen begleitet wird, deren Trennung und nähere Charakterisierung den Gegenstand weiterer Mitteilungen bilden soll. Die Versuche werden gleichzeitig auch in synthetischer Richtung geführt. 